\title{
Temporoparietal Fascia Free Flap for a Large Nasal Septal Perforation
}

\author{
Nicholas G. Cuccolo, BS ${ }^{1}$ Ryan P. Cauley, MD ${ }^{1}$ Brady A. Sieber, MD ${ }^{1}$ Syed M.D. Hussain, MD \\ Laurel K. Chandler, MD ${ }^{1}$ Ahmed M.S. Ibrahim, MD, $\mathrm{PhD}^{1} \quad$ Samuel J. Lin, MD, MBA ${ }^{1}$
}

1 Division of Plastic and Reconstructive Surgery, Beth Israel Deaconess
Medical Center, Harvard Medical School, Boston, Massachusetts
2Baim Institute for Clinical Research, Boston, Massachusetts

J Reconstr Microsurg Open 2019;4:e42-e46.
Address for correspondence Samuel J. Lin, MD, MBA, Division of Plastic and Reconstructive Surgery, Beth Israel Deaconess Medical Center, Harvard Medical School, 110 Francis Street, Suite 5A, Boston, MA 02215 (e-mail: sjlin@bidmc.harvard.edu).

\begin{abstract}
Keywords

- nasal septum reconstruction

- temporoparietal fascia flap

- microvascular free tissue transfer

Reconstruction of large nasal septal perforations can be a considerable challenge for surgeons. Reconstructive modalities are often determined by the size of the defect and the quality of local tissue and vasculature. Local and regional flaps may not provide enough tissue to achieve successful closure in patients with large perforations and those with baseline compromise of the nasal mucosa and blood supply from prior intervention or underlying medical condition. Microvascular free tissue transfer is a possible approach to reconstruction in these patients. We report a case of a 30-year-old man who presented with a large, symptomatic, $4 \times 3.5 \mathrm{~cm}$ perforation as a result of prior functional septoplasty at an outside hospital. Reconstruction of the defect was accomplished in this setting with a free temporoparietal fascia (TPF) flap anastomosed to the columellar branch of the labial artery and the angular vein. Postoperative followup visits showed successful closure of the perforation without complications. As illustrated by this case, TPF flap is a versatile tool for complex nasal reconstructions. With minimal donor-site morbidity and rich vascularity capable of promoting remucosalization without the need for prelamination, the TPF flap may be suited for the repair of large nasoseptal perforations.
\end{abstract}

Defects of the nasal septum represent a unique challenge to surgeons, given its location, complex tissue structure, and functional and aesthetic roles of the nasal septum. Furthermore, local tissue and blood supply may be compromised as a result of prior surgery, which is the most common etiology of perforation. ${ }^{1}$ Experiencing the loss of a functional mucociliary apparatus and the disruption of physiologically laminal airflow, patients often report symptoms such as nasal obstruction, dryness/crusting, whistling, and paranasal pain. Moreover, loss of the structural support afforded by the nasal septum can lead to cosmetic defects including dorsal nasal saddling, columellar retraction, and dropping of the nasal tip. ${ }^{1}$

Numerous methods of reconstructing the nasal septum have been reported with varying degrees of success, yet no consensus on the optimal technique currently exists. ${ }^{2}$ Frequently, perforations less than $2.0 \mathrm{~cm}$ in diameter are amenable to closure with local mucosal flaps or even allograft or synthetic materials such as dermal substitutes $^{3}$ or bioactive glass. ${ }^{4}$ However, symptomatic defects larger than $2.0 \mathrm{~cm}$ often require a greater amount of vascularized tissue to achieve adequate closure, thus making regional or free flaps the preferred option in these cases. $^{5}$

The temporoparietal fascia (TPF) flap, with its thin and pliable structure, reliable arterial and venous pedicle, and consistent vascular anatomy, ${ }^{6}$ is one of the most versatile flaps in the body. The utility of the TPF flap has been demonstrated for coverage in a myriad of situations ${ }^{7}$ and is also an excellent option for the reconstruction of large received

April 4, 2019

accepted

April 15, 2019
DOI https://doi.org/

10.1055/s-0039-1692145. ISSN 2377-0813.
Copyright $\odot 2019$ by Thieme Medical

Publishers, Inc., 333 Seventh Avenue, New York, NY 10001, USA. Tel: +1(212) 584-4662.
License terms

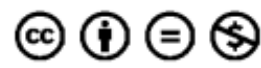




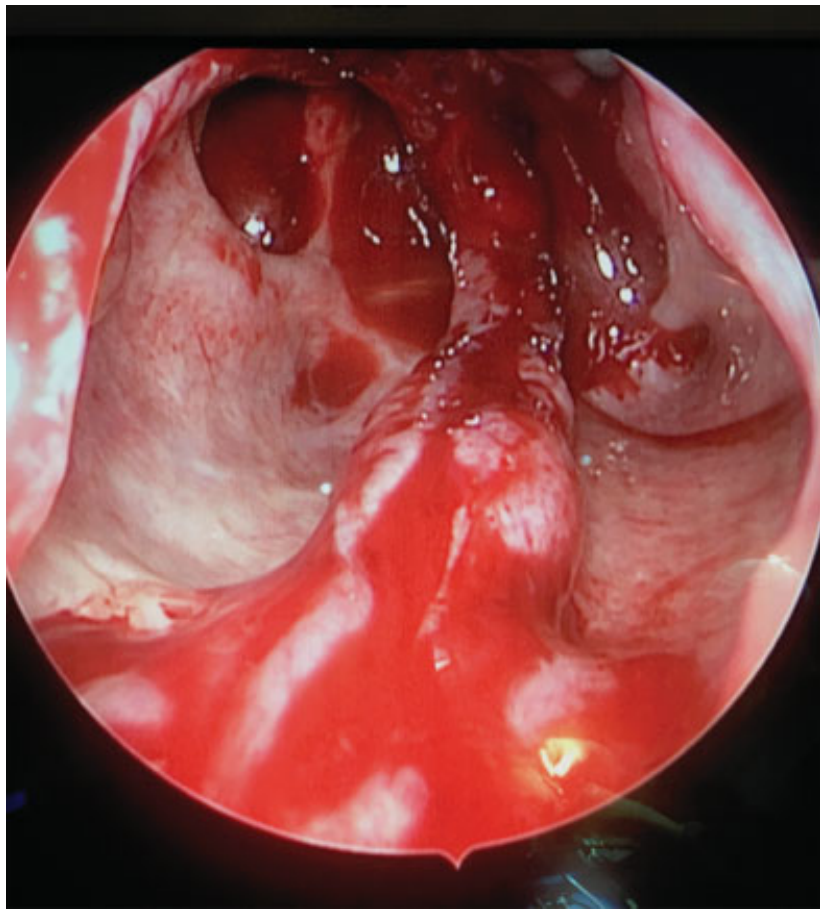

Fig. 1 Large perforation on preoperative nasal endoscopy.

nasoseptal defects. We present a case describing the use of a TPF free flap for repair of a large septal perforation.

\section{Case Details}

A 30-year-old male with no past medical or substance use history originally presented to the clinic with chronic nasal obstruction following a prior functional nasal surgery at an outside hospital that was complicated by bleeding and a large septal perforation. Endoscopic examination revealed a $4.0 \times 3.5 \mathrm{~cm}$ chronic perforation (-Fig. 1), consistent with prior computed tomography (CT) scans (-Fig. 2). Options for the reconstruction of the perforation were evaluated, including local intranasal mucosal flaps, synthetic dermal substitutes, regional flaps, and free tissue transfer. Given the size of the defect,

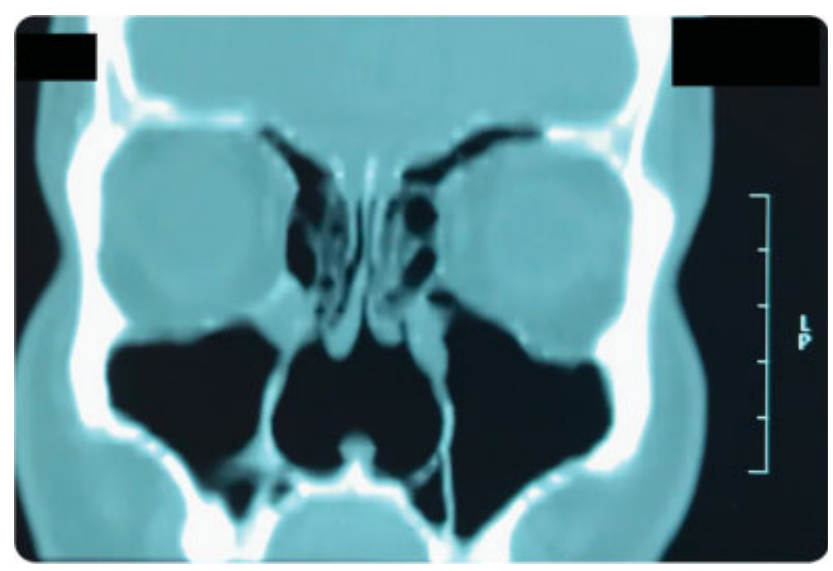

Fig. 2 Prior computed tomography (CT) scan revealing large nasoseptal defect.

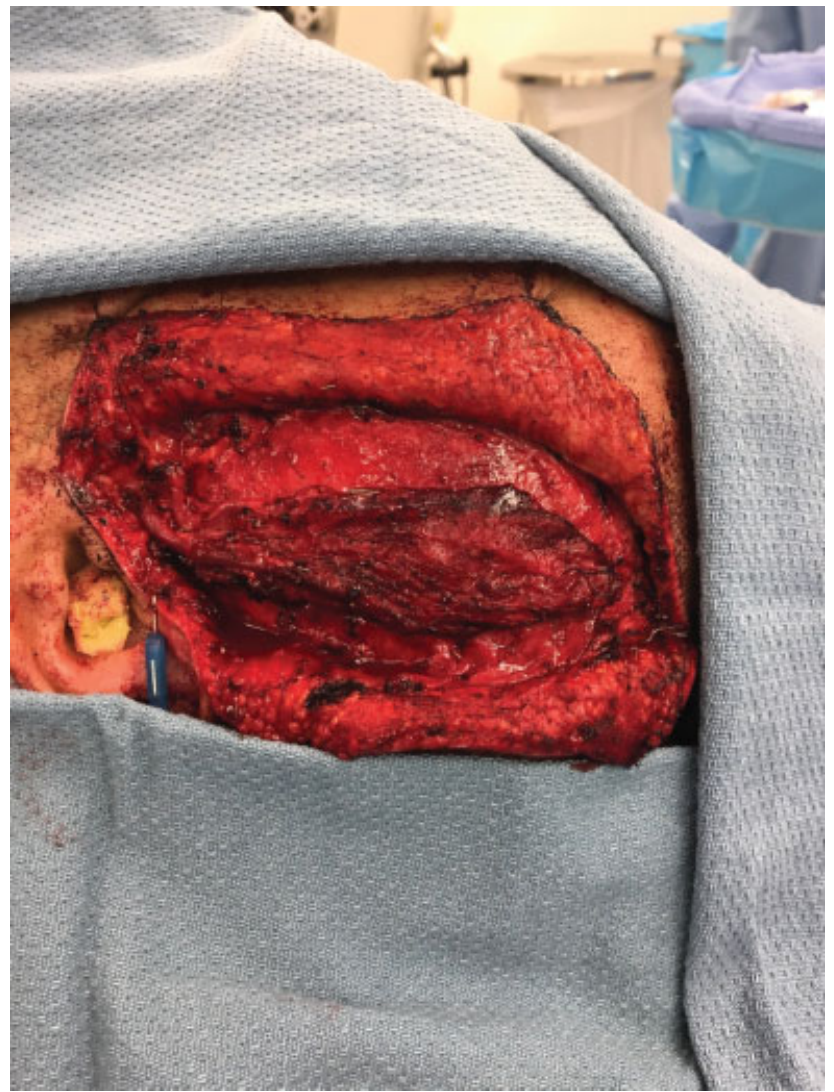

Fig. 3 Harvest of the temporoparietal fascia free flap.

history of prior nasal surgery, and the dearth of local options, vascularized free tissue transfer was deemed the most appropriate reconstructive modality. Regarding donor-site options, the TPF free flap was selected for septal repair due to its thin and pliable structure. Additionally, the exceptional vascularity of the TPF enabled a potential reconstruction without the need for prelamination or use of interposition grafts.

Preoperatively, the superficial temporal artery (STA) and the angular artery were identified using a Doppler probe. Dissection of the STA and the superficial temporal vein (STV) proceeded through a preauricular incision. A fascial flap measuring $9 \times 5 \mathrm{~cm}$ was designed and harvested ( - Fig. 3 ) with a vascular pedicle. Exposure of the nasal septum was obtained through left-sided limited lateral rhinotomy and nasolabial incisions.

The left angular artery and vein were dissected through the initial incisions. No suitable branches of the angular artery were identified, and the columellar branch of the superior labial artery was dissected (-Fig. 4). Circumferential flaps were raised around the septal perforation, and the TPF flap was brought to the ipsilateral cheek area. Venous anastomosis between the STV and a branch of the angular vein was accomplished using a $2.5-\mathrm{mm}$ coupling device (-Fig. 5). The STA was then anastomosed to the columellar branch of the superior labial artery, which measured approximately $0.8 \mathrm{~mm}$, in an end-to-end fashion. A 2:1 donor to recipient vessel size mismatch was overcome using a coning technique on the larger vessel. 


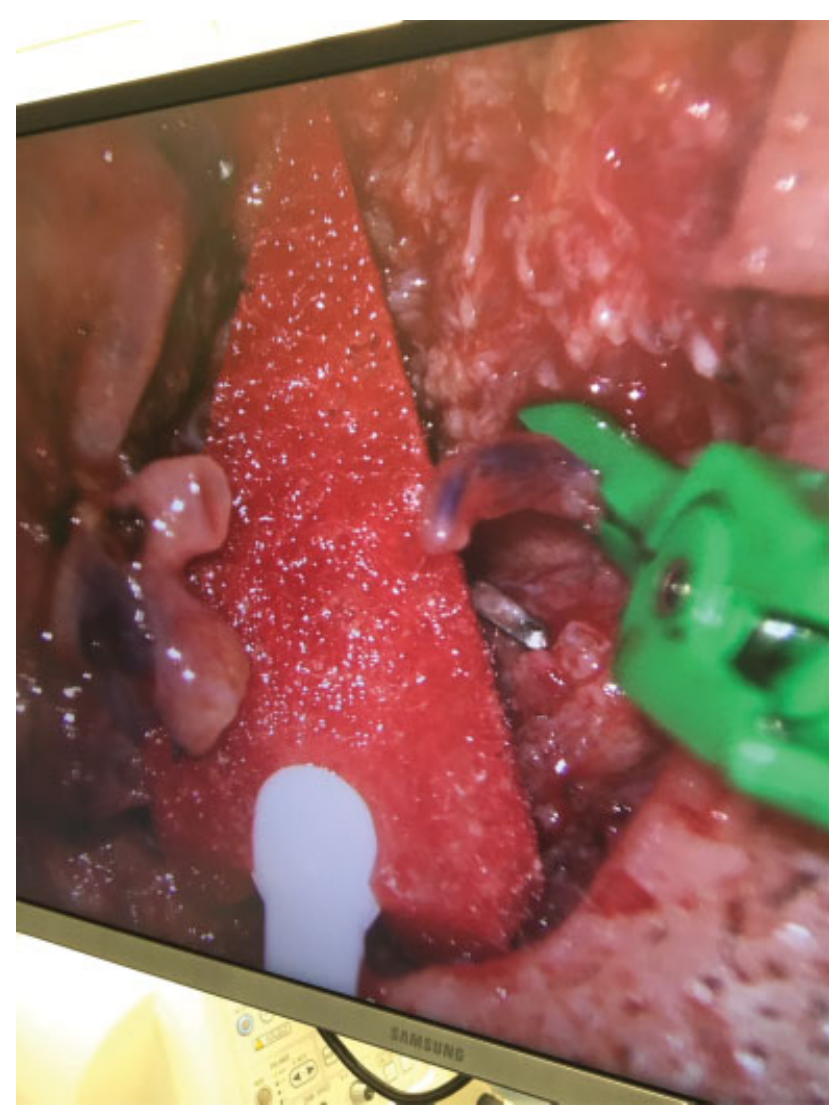

Fig. 4 The vessel being held by the green clamp is the columellar branch of the superior labial artery. The vessel on the left (lumen can be visualized) is the superficial temporal artery).

Using an endoscope placed in the contralateral nares, the flap was then parachuted into place and inset with 3-0 Vicryl horizontal mattress sutures (-Fig. 6). Given the small caliber of the piriform aperture, knots were tied with the assistance of a laparoscopic knot pushing device. Confirmation of successful closure of the perforation was accomplished endoscopically. Skin was then reapproximated over a drain, and nasal packing was placed. Postoperative monitoring of the flap with a handheld Doppler probe revealed strong arterial and venous signals. The remainder of the postoperative course proceeded without complications, and the patient was discharged on postoperative day 4. Follow-up examination at 2 months revealed successful closure of the perforation and complete mucosalization of the flap (-Fig. 7). The donor site was without complications, and the patient reported resolution of symptoms.

\section{Discussion}

Reconstructive modalities for nasal septal perforations vary depending on the size and location of the defect, symptomatology, quality of local tissue, and surgeon experience. Small, posteriorly located perforations are often asymptomatic, are a byproduct of the prompt humidification of inspired air through the nasal mucosa and turbinates, ${ }^{2}$ and do not warrant treatment. Anterior perforations, in comparison, are more likely to distort physiologic airflow dynamics,

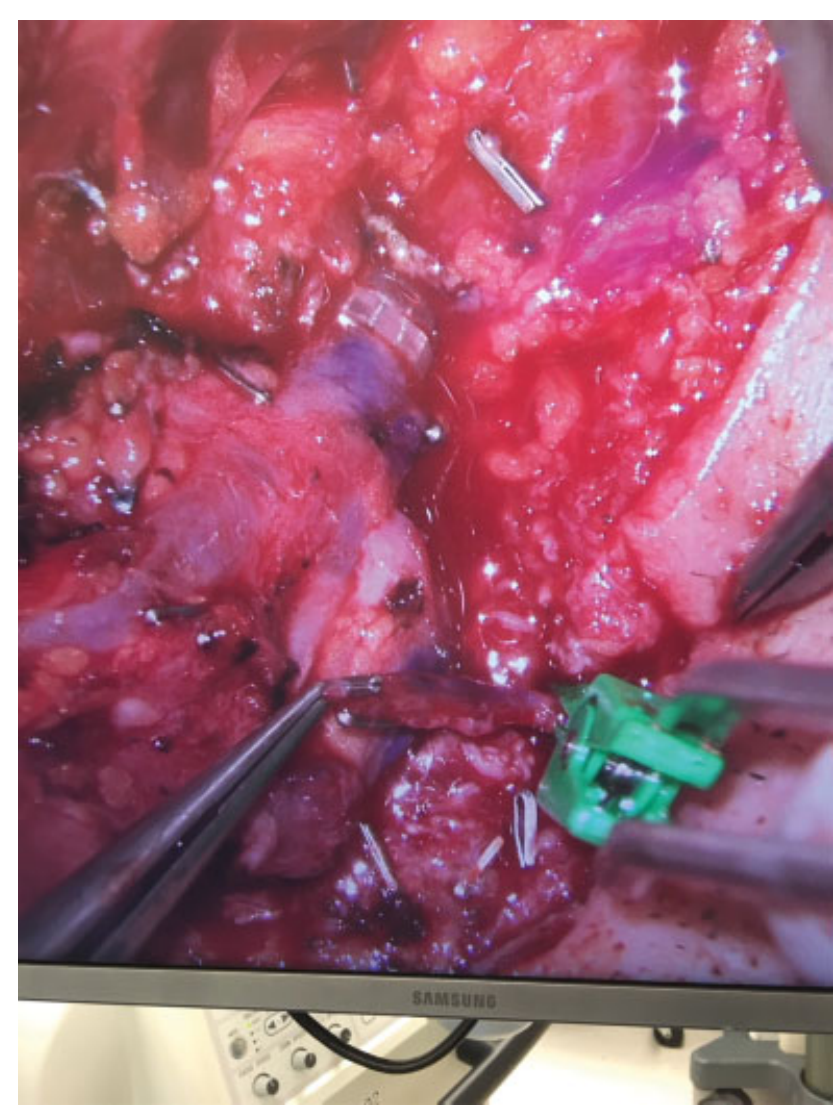

Fig. 5 Venous anastomosis through a 2.5-mm coupler of the superficial temporal vein to the angular vein.

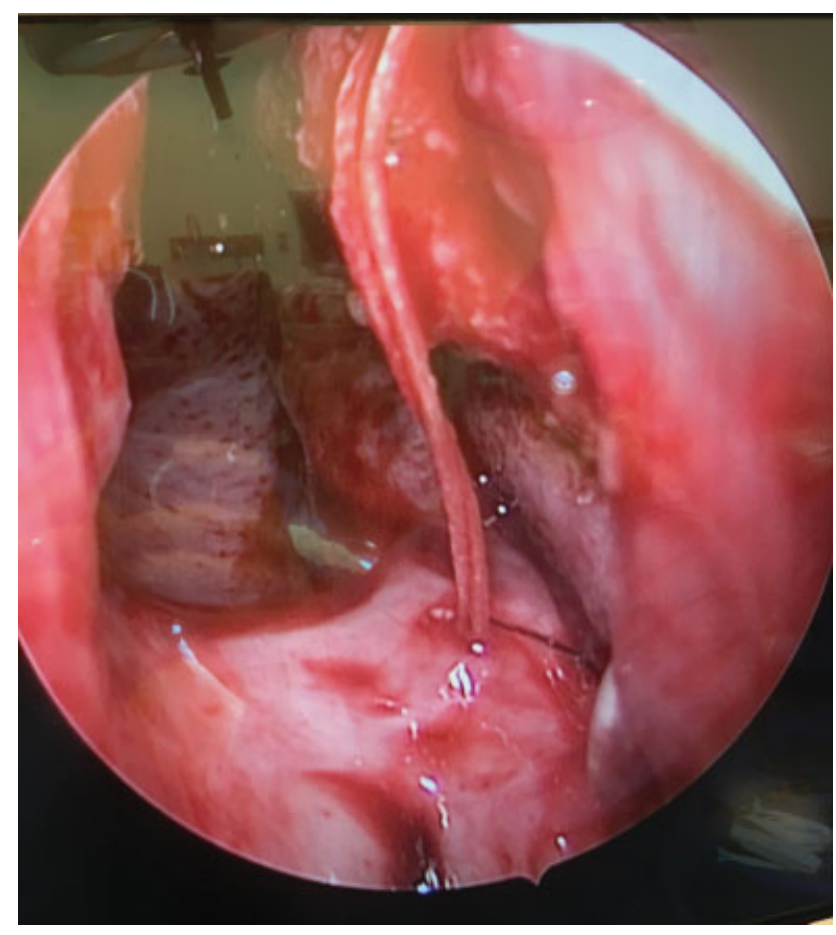

Fig. 6 Intranasal temporoparietal fascia flap inset. 


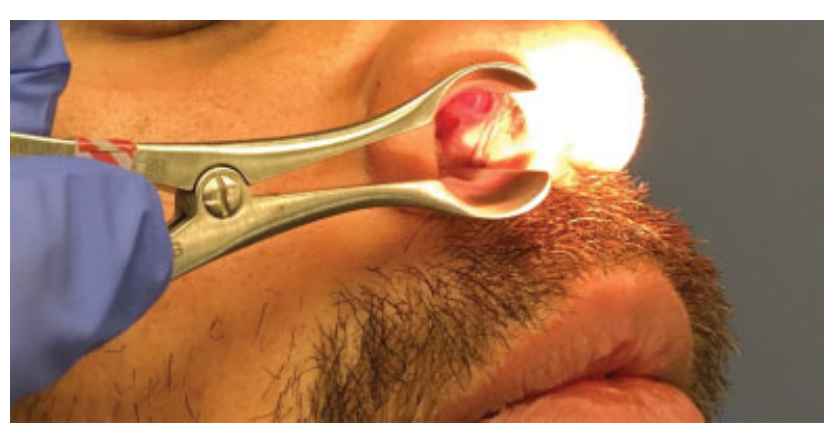

Fig. 7 Two-month postoperative follow-up examination showing closure of the perforation and mucosalization of the flap.

thus producing symptoms of obstruction, whistling, and pain. Smaller, symptomatic perforations may be amenable to long-term medical therapy with nasal saline irrigation, topical antibiotics, and humidified air. ${ }^{8}$

Persistence of symptoms despite optimal medical therapy frequently necessitates surgical intervention. Intranasal mucosal flaps, with or without interposition grafts, are often used for smaller perforations and have reported success rates of 90 to $100 \%{ }^{5}$ Given that the amount of mucosa available for closure is inversely related to the size of the defect, the surgeon's ability to achieve a tension-free approximation becomes more challenging with larger perforations. Tissue expansion has been described as a means of circumventing this issue, ${ }^{9}$ but the need for multiple procedures is a significant drawback. Furthermore, a common etiology of septal perforation is prior nasal surgery itself, ${ }^{1}$ which can be associated with scarring and compromise of local blood supply, thereby limiting the utility of intranasal flaps. As such, regional flaps, including the facial artery musculomucosal flap ${ }^{5}$ and the pericranial flap, ${ }^{10}$ have been used in the closure of more sizeable defects.

In the context of even larger perforations, such as those greater than $3.5 \mathrm{~cm}$, successful closure rates have been reported at $70 \%{ }^{1}$ For these patients, local and regional options may be inadequate. The advent of free tissue transfer significantly expanded the available reconstructive modalities in the head and neck. Regarding nasoseptal reconstruction in particular, the free radial forearm flap ${ }^{11}$ and the fascia lata free flap ${ }^{12}$ have been reported in the literature with favorable results.

Although the utility of the radial forearm flap in head and neck reconstruction has been well documented, ${ }^{6}$ the bulky nature of this tissue often necessitates thinning when used in septal repair. Moreover, there is considerable donor-site morbidity, which must be taken into consideration when evaluating potential reconstructive modalities. For these reasons, a free fascial flap such as the TPF may be a more ideal option for septal reconstruction. Not only is the TPF flap considerably thinner than other free tissue options, but it can also be harvested with minimal donor-site morbidity using incisions often placed posterior to the hairline.

Extending cephalad from the superficial musculoaponeurotic systemic in the face, the TPF is located over the temporal fossa, spanning from the zygomatic arch to the vertex. ${ }^{6}$ Based on the STA and STV, which often range from
2.5 to $5.0 \mathrm{~cm}$ in length, the TPF is often harvested with dimensions of $14 \times 12 \mathrm{~cm}$, although this can be extended through tissue expansion. ${ }^{6}$ As a thin and malleable flap with rich vascularity, the TPF flap is well suited for the repair of complex nasal defects and has been described for reconstruction of nasal lining, skin cover, and septum. ${ }^{13-15}$

As this case highlights, the TPF flap imparts minimal donor-site morbidity and leaves a relatively inconspicuous scar, which is especially important in patients who are concerned with the cosmetic outcome. In comparison to the often-bulky radial forearm flap, the TPF flap is significantly thinner and thus obviates the potential need for debulking. Although the benefits of prelamination in fascial flaps have been described, ${ }^{12}$ the dependable vascularity of the TPF flap facilitates remucosalization by secondary intention, typically within 3 weeks. Ultimately, this precludes a prelamination procedure.

This case report demonstrates the utility of the TPF free flap for nasal septal reconstruction. This technique is useful in patients with larger defects or those who have limited local options as a result of prior surgery, trauma, cocaine abuse, or other disease processes. Given the inconspicuous donor-site scar and the ability to achieve tension-free closure, the TPF flap may be considered for the repair of large septal defects.

\section{Ethical Approval}

Consent for the inclusion of patient information and photographs for purposes of research and publication was obtained prior to composition of this manuscript. Institutional review board approval was not required for this case report in accordance with the Department of Health and Human Services' definition of human research subjects.

\section{Note}

Portions of this work were presented at the Mountain West Society of Plastic Surgeons Annual Scientific Meeting in Olympic Valley, CA, March 7-10, 2019.

\section{Disclosure}

None of the authors have a financial interest in any of the products, devices, drugs, or procedures mentioned in this manuscript.

\section{Funding}

There was no internal or external financial support for this study.

\section{Conflict of Interest}

None declared.

\section{References}

1 Foda HM, Magdy EA. Combining rhinoplasty with septal perforation repair. Facial Plast Surg 2006;22(04):281-288

2 Goh AY, Hussain SS. Different surgical treatments for nasal septal perforation and their outcomes. J Laryngol Otol 2007;121(05): 419-426

3 Ayshford CA, Shykhon M, Uppal HS, Wake M. Endoscopic repair of nasal septal perforation with acellular human dermal allograft 
and an inferior turbinate flap. Clin Otolaryngol Allied Sci 2003;28 (01):29-33

4 Stoor P, Grénman R. Bioactive glass and turbinate flaps in the repair of nasal septal perforations. Ann Otol Rhinol Laryngol 2004;113(08):655-661

5 Heller JB, Gabbay JS, Trussler A, Heller MM, Bradley JP. Repair of large nasal septal perforations using facial artery musculomucosal (FAMM) flap. Ann Plast Surg 2005;55(05):456-459

6 Wei FC, Mardini S. Flaps and Reconstructive Surgery E-Book. Philadelphia, PA: Elsevier Health Sciences; 2016

7 Brent B, Upton J, Acland RD, et al. Experience with the temporoparietal fascial free flap. Plast Reconstr Surg 1985;76(02):177-188

8 Coleman JR Jr, Strong EB. Management of nasal septal perforation. Curr Opin Otolaryngol Head Neck Surg 2000;8(01):58-62

9 Romo T III, Jablonski RD, Shapiro AL, McCormick SA. Long-term nasal mucosal tissue expansion use in repair of large nasoseptal perforations. Arch Otolaryngol Head Neck Surg 1995;121(03): 327-331
10 Paloma V, Samper A, Cervera-Paz FJ. Surgical technique for reconstruction of the nasal septum: the pericranial flap. Head Neck 2000;22(01):90-94

11 Mobley SR, Boyd JB, Astor FC. Repair of a large septal perforation with a radial forearm free flap: brief report of a case. Ear Nose Throat J 2001;80(08):512

12 Bank J, Beederman M, Naclerio RM, Gottlieb LJ. Prelaminated fascia lata free flap for large nasal septal defect reconstruction. J Plast Reconstr Aesthet Surg 2014;67(10):1440-1443

13 Cinpolat A, Bektas G, Coskunfirat OK. Complex partial nasal reconstruction using free prelaminated temporoparietal fascial flap. Microsurgery 2013;33(02):156-159

14 Acikel C, Bayram I, Eren F, Celikoz B. Free temporoparietal fascial flaps and full-thickness skin grafts in aesthetic restoration of the nose. Aesthetic Plast Surg 2002;26(06):416-418

15 Helman S, Kadakia S, Guthrie A, Mourad M, Mashkevich G. Temporoparietal fascia free flap for nasoseptal perforation repair. Craniomaxillofac Trauma Reconstr 2018;11(03):238-241 